\title{
The Origin of High Energy Variability in Blazars
}

\section{lan $\mathbf{M}^{\mathrm{c}}$ Hardy*}

University of Southampton, $U K$

E-mail: imhesoton.ac.uk

I present a brief, and slightly rough, summary of my talk at the May 2011 Toulouse CTA meeting which deals with the origin of X-ray and Gamma-ray variability in blazars such as 3C273 and $3 \mathrm{C} 279$

For full details please see http://cta.obspm.fr/agnworkshop2011/presentations/MONDAY/cta_agn_mchardy.pdf which is largely self-explanatory. If time permits I may update this contribution later.

The main observational conclusions are that, in the X-ray band, blazars behave very much like Seyfert galaxies (ie non-beamed AGN). They show rms-flux relations and have bends in their power spectral densities (PSDs) whose timescales are consistent with Seyfert mass/accretion rate scaling. The implication is that the perturbations which are thought to propagate inwards through the accretion disc in Seyfert galaxies to modulate the X-ray emitting corona also modulate the $\mathrm{X}$-ray emission region in blazars. The Gamma-ray lightcurves are, however, very different to those in the X-ray band. There are a small number of brief flares which are seen in both bands but the bulk of the variability is different, implying at least two separate emission regions in both bands. The flares seen in both bands may arise from shocks in a relativistics jet, and could occur far from the black hole. The Gamma-ray PSDs have slopes (-1) similar to that of the X-rays at low frequencies but cannot yet confirm or deny the existence of bends. If bulk of the X-ray and Gamma-ray emission comes from a steady jet, with the Gamma-ray emission arising closer to the black hole, it is hard to see how PSD bends could be imprinted on the X-ray emission without also being imprinted on the Gamma-ray emission unless the variability is primarily produced within the jet rather than propagating in, through the corona, from the accretion disc. Hopefully further Fermi data will eventually clarify the important question of whether blazar Gamma-ray PSDs have bends at the same frequencies as in the X-ray band.

Some of this work has been done in collaboration with Alan Marscher, Svetlana Jorstad, Alex Markowitz, Dimitrios Emmanoulopoulos and others, but I don't blame them for the brevity of this contribution.

AGN Physics in the CTA Era - AGN2011,

May 16-17, 2011

Toulouse, France

${ }^{*}$ Speaker. 


\section{The Important Questions in Blazar Variability}

When trying to understand blazar variability there are a number of topics which we should consider:

- Are blazar lightcurves produced by the summation of some sort of special shots or outburts, or are they simply the result of a noise process?

- Can we learn anything by comparison with the well-studied X-ray variability of X-ray binaries (XRBs) or Seyfert galaxies, ie non-beamed AGN.

- What can we learn about the emission process and source of the variations by studying the relationship between the variations in different bands, eg X-ray and Gamma-ray?

- Is blazar variability dominated by processes within the jet (eg turbulence) or outside the jet (eg accretion disc perturbations); ie does the host black hole leave any imprint on the observed Gamma-ray variability?

\section{Independent flares or just a noise process? What can we learn from X-ray binaries and Seyfert galaxies?}

Blazar observers usually like to concentrate on periods when there is a rapid rise in flux. These periods are often referred to as flares or outbursts. But are these outbursts special events, or are they just part of a stochastic noise process? As an example I show, in Fig.1, the X-ray lightcurve of 3C279 decomposed into a number of separate flares by Chatterjee et al [1]. This decomposition works perfectly well, but is it the truth? I also show, an example of a flare in 3C454.3 which is particularly prominent in the Gamma-ray band, but where flux rises are also seen in other bands ([纤).
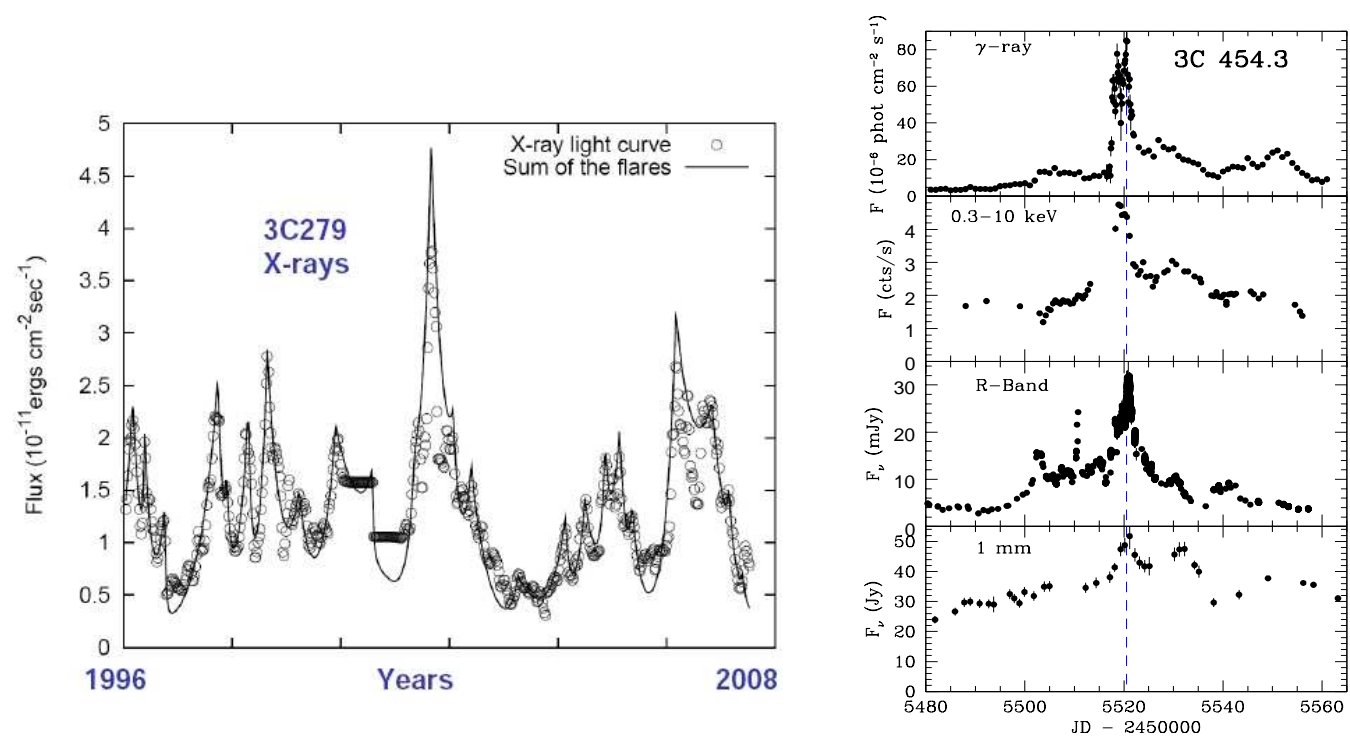

Figure 1: Left: X-ray lightcurve of 3C279 decomposed into individual flares by [1]]; Right: An example of a large 'flare', particularly prominent in the Gamma-ray band, as observed in a number of different wavebands ([2]) 


\subsection{Lessons from X-ray Binaries and Seyfert Galaxies}

Rapid X-ray variability is seen in X-ray binary systems (XRBs) and in non-beamed AGN, ie Seyfert galaxies (eg [3]). One strong characteristic of their variability is that there is a strong relationship between the rms variability at a given time and the mean flux level ([7]). This relationship tells us that the short timescale variations are coupled to those on longer timescales. A possible cause of the variations could be the propagation inwards of accretion rate variations through the accretion disc with longer timescale variations being produced at larger radii. A linear rms-flux relationship will not be produced by a random distribution of independent flares. Such a distrubution will tend to produce a constant rms.

In Figs. 2 and 3 I show the rms-flux relationship for the X-ray variations in the blazars 3C273 and 3C279 ([6]) in preparation). Both blazars show the same relationship that we see in XRBs and Seyfert galaxies. These observations argue against an interpretation of blazar variability as being due to a series of entirely independent flares or shots.

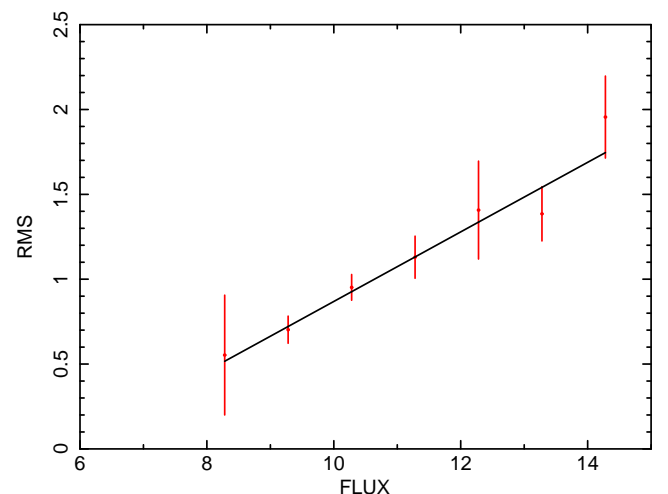

Figure 2 :rms-flux relationship for the $\mathrm{X}$-ray variations in $3 \mathrm{C} 273$.

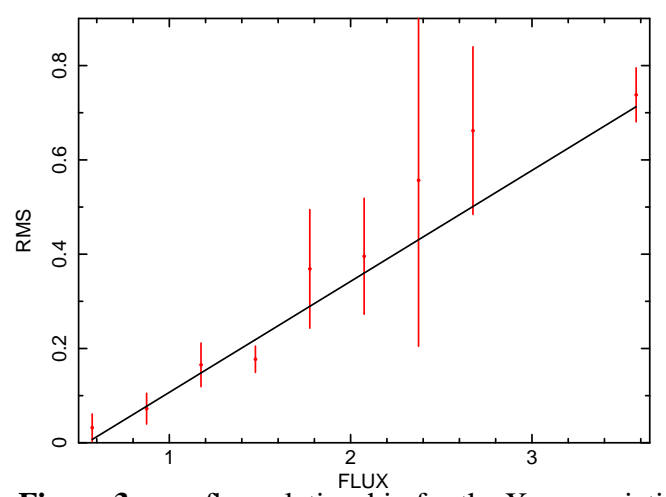

Figure 3 :rms-flux relationship for the $\mathrm{X}$-ray variations in $3 \mathrm{C} 279$.

\section{The relationship between variations in different wavebands}

I concentrate here on the relationship between the X-ray and Gamma-ray bands. As a result of the long timescale X-ray monitoring programmes carried out with the Rossi X-ray Timing Explorer (RXTE) by Alan Marscher, Svetlana Jorstad, myself and others it is now possible to make excellent long timescale X-ray lightcurves. Similarly the FERMI database now enables one to make excellent long timescale Gamma-ray lightcurves.

In Figs.4 and 6 I show parallel X-ray and Gamma-ray lightcurves for 3C273 and 3C279 covering periods of 3 years with time resolution of a few days. We see that there is actually very little correlation between the wavebands. Occasionally, when there is a very large 'flare' in one waveband, it is seen in the other (see Fig.5 for one such event), but not always. The correlation is quantified in Figs. 7 and 8 where I show the cross-correlation functions. In each case there is a very weak correlation at close to zero lag.x This part of the correlation results from the few parallel flares (eg Fig.5 and see Fig.1 for a similar event). However the bulk of the variations in the two bands are different. I conclude that there are at least two components to the high energy variability in blazars in any given waveband. One of these components is associated with a physically localised event, where emission is produced over a wide range of wavebands. This event could well be a strong shock, which would give rise to frequency-stratified emission and to the very small lags 
between wavebands which are seen in more detailed multiband studies of 'flares' (eg Fig.1, from [2]. However the bulk of the emission in the X-ray and Gamma-ray bands appears to come from regions which are physically separated enough that perturbations originating in one region do not noticeably affect variability in the other emission region.

It is interesting to compare the relationship between the X-ray and Gamma-ray emission, which we see here to be quite weak, and the relationships between the X-ray and lower energy emissions in blazars which are generally stronger. For example, reasonable correlations are found between X-ray and both optical and radio variability in 3C279 (see [1] for more details). We return to this point in the brief conclusions section.

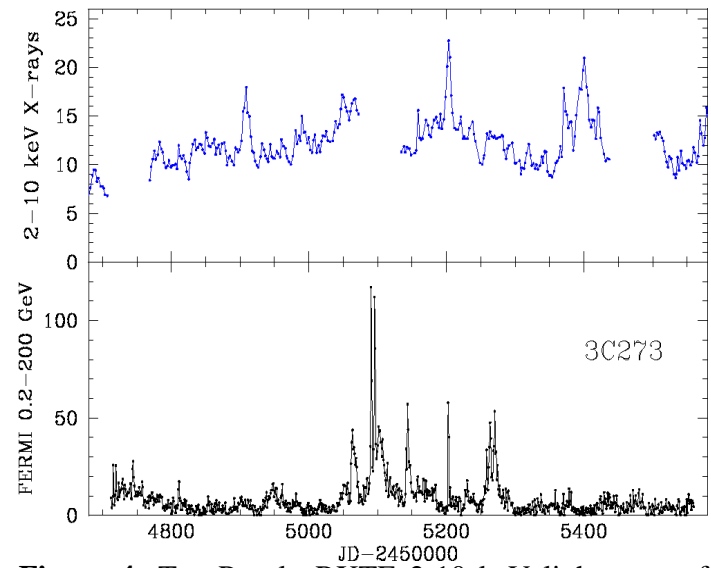

Figure 4 :Top Panel: RXTE 2-10 keV lightcurve of 3C273. Bottom Panel: Fermi 0.2-200 GeV Gamma-ray lightcurve. With the possible exception that the major period of Gamma-ray activity occurs when the X-ray mean flux is fractionally higher, there is very little correlation between the two wavebands.

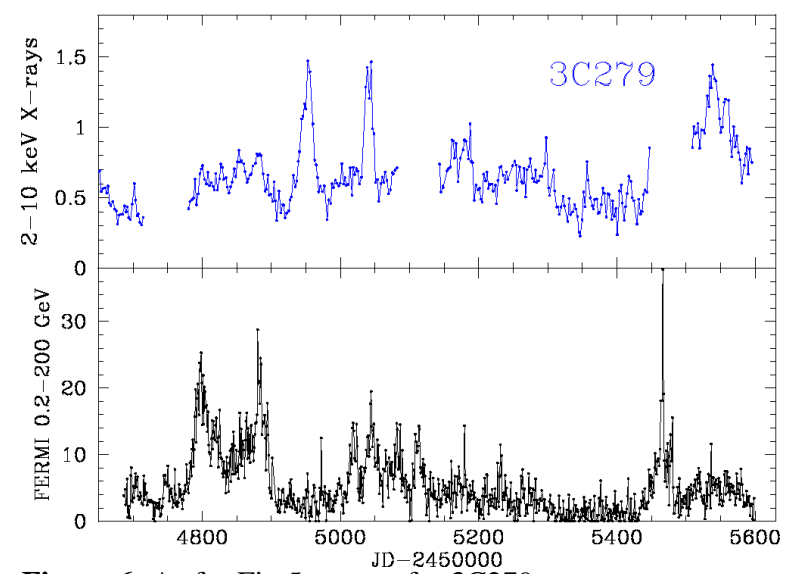

Figure 6 :As for Fig.5, except for 3C279.

\section{Power Spectra}

We conclude this brief data review by showing the X-ray and Gamma-ray power spectral densities (PSDs) of 3C273 and 3C279 (from [6]). From Fig.9 we can see a clear bend in the long

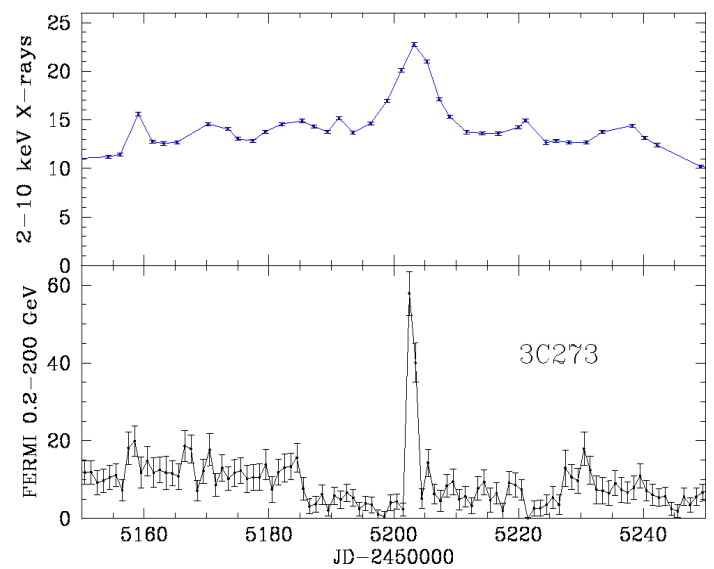

Figure 5 :Similar to Fig.5 except zooming in on a smaller time period, showing one correlated flare in both bands. 


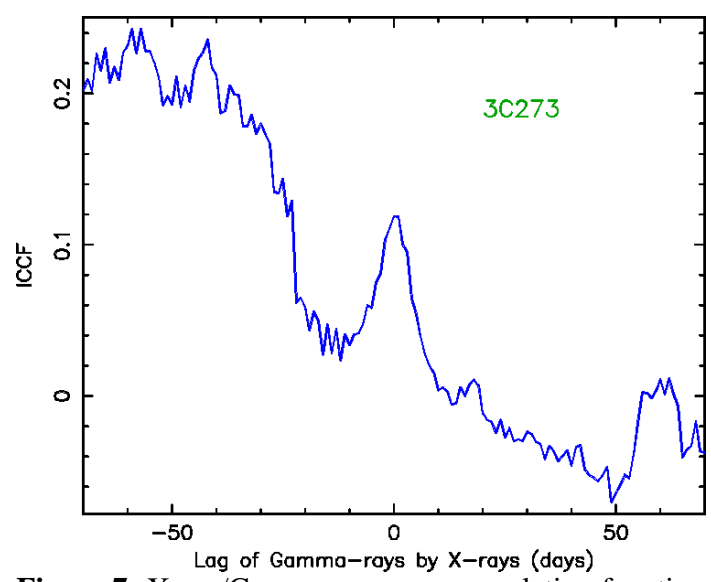

Figure 7 :X-ray/Gamma-ray cross correlation function for 3C273 from the lightcurves shown in Fig.5.

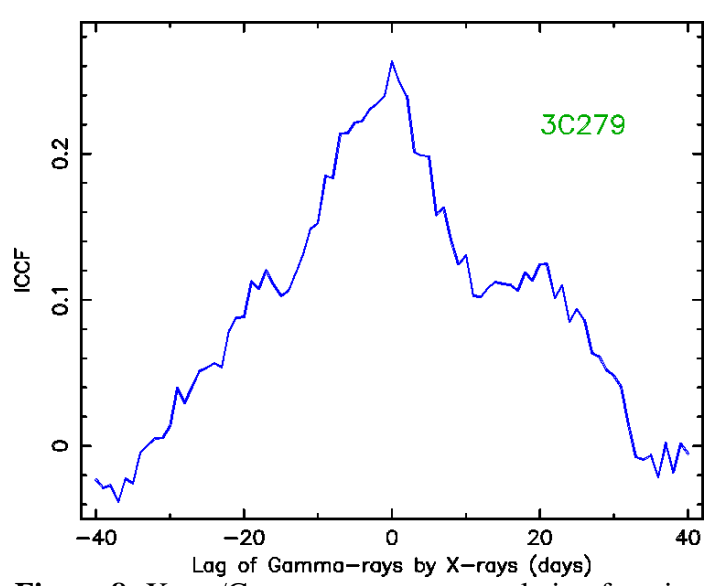

Figure 8 :X-ray/Gamma-ray cross correlation function for 3C279 from the lightcurves shown in Fig.7.

timescale X-ray PSD of 3 C273. The bend frequency is consistent with the scaling relationship between bend timescale, black hole mass and accretion rate for Seyfert galaxies and soft-state XRBs (沛). The Fermi data do not cover the high frequencies very well, and the data are less extensive and so the quality of the PSD is poorer, thus we cannot draw strong conclusions. The slope in the measured frequency range is -1, the same as for the X-ray emission in the same (low) frequency range. However there is no strong evidence for a bend in the Gamma-ray PSD within the present data. (Note that the ordinates in Figs 7 and 8 are, for various technical reasons, in different units.)

For 3C279 we can update the X-ray PSD provided by [1] with additional data (Fig.9). We now see a clear bend at a frequency of $\sim 3 \times 10^{-7} \mathrm{~Hz}$. In the Fermi PSD the result is less clear. The slope (-1) is the same as in the X-ray band, in the same (low) frequency range, and the PSD is consistent with a bend at the same frequency. However further data are required to confirm the reality of the bend in the Fermi PSD.

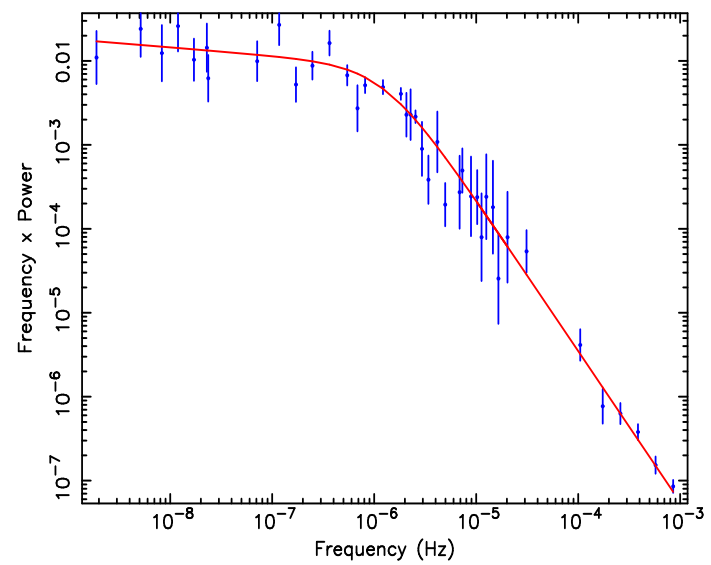

Figure 9 :X-ray power spectrum of $3 \mathrm{C} 273$ with combined data from many observatories $\left(\mathrm{M}^{\mathrm{c}}\right.$ Hardy et al, in preparation). Note that the ordinate is units of frequency* power.

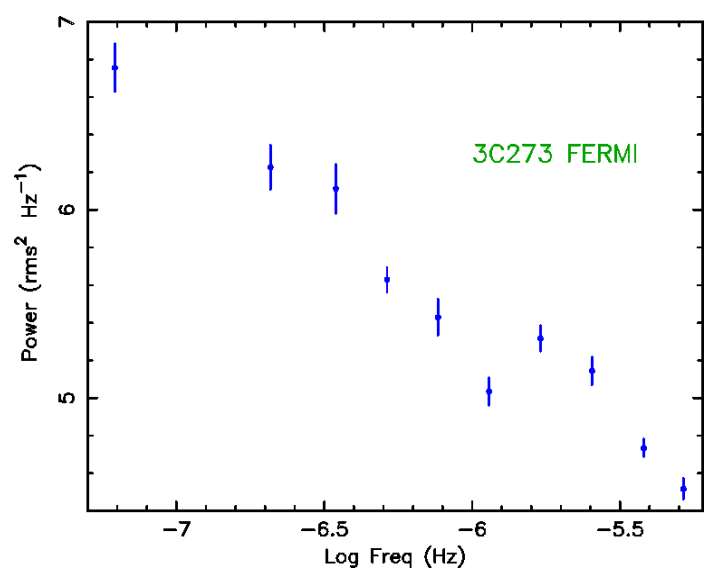

Figure 10 : Fermi Gamma-ray power spectrum of 3 C273. Here the ordinate is in units of just power. 


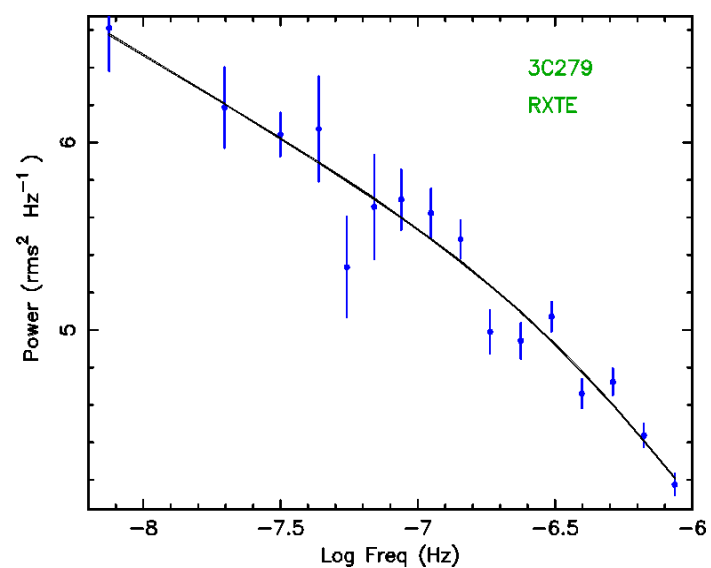

Figure 11 : RXTE X-ray power spectrum of 3C279 ( $\mathrm{M}^{\mathrm{c}}$ Hardy et al, in preparation). The ordinate is units of power.

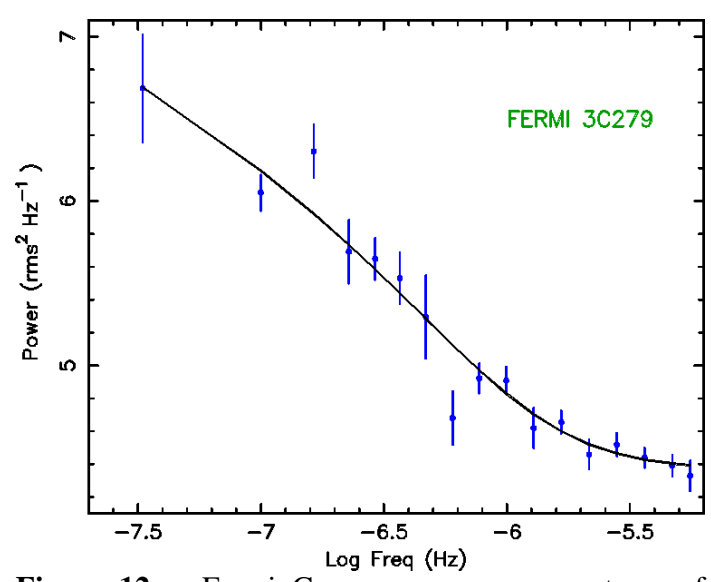

Figure 12 : Fermi Gamma-ray power spectrum of $3 \mathrm{C} 279$. The ordinate is in units of power.

\section{Conclusions}

The weak correlation between the X-ray and Gamma-ray bands tells us that there are at least two sources of variability in both wavebands. Shocks, which may be far removed from the black hole, in a jet may account for the flares which are seen in both bands, but how do we account for the bulk of the variability?

In the X-ray band, the variability characteristics of blazars are similar to those of Seyfert galaxies (ie non-beamed AGN) and XRBs. They show similar rms-flux relationships and bends are found in their PSDs which fit the same mass/accretion rate scaling relationships as those found in Seyferts and XRBs. These observations indicate that, at least as far as the bulk of the X-ray emission goes, the central black hole leaves an imprint upon the emission characteristics. In Seyfert galaxies it is commonly assumed that perturbations arising in the accretion disc propagate inwards and modulate the $\mathrm{X}$-ray emission from some central corona. In blazars we might therefore say that the X-ray emission also comes from a central corona. However observations such as the strong correlation between the X-ray and infrared emission in 3C273 [5], and the fact that the X-ray lag the infrared by about a day, strongly implies that the bulk of the X-ray emission is produced by synchrotron self-Compton emission, ie from a jet, rather than from thermal Compton emission from a non-beamed central corona. In that case we would conclude that the perturbations carry on through any central corona into a jet which would extend out from that corona.

In the Gamma-ray band the conclusions are less clear. The PSD slopes are the same as at low frequencies in the X-ray band (-1) and there is a hint of a bend at the same frequency in $3 \mathrm{C} 279$, but the evidence is far from overwhelming at present. It is hard to produce high luminosity Gamma-ray emission from anything other than a relativistic jet oriented towards the observer. In such a jet then, apart from in strong shocks which could arise far from the black hole, the bulk of the Gamma-ray emission arises close to the black hole where particle energies and magnetic field strengths are highest. If we assume that the Gamma-ray and X-ray emissions both come from a relativistic jet, with the X-ray emission arising further from the black hole, and we further assume that the perturbations propagate down the jet, then it is very hard to see how the imprint of the 
black hole upon those variations can somehow bypass the Gamma-ray emission region but still affect the downstream X-ray emission region. If the PSD bend is not present, or is at a much higher frequency than in the X-ray band, we must conclude that the underlying source of the variability in the Gamma-ray band is not external to the emission region, eg from the disc, but is produced mainly by processes within the jet. Hopefully, once further Fermi data have been accrued, the very important question of whether there is a bend in the Gamma-ray PSD can be clarified.

\section{References}

[1] Chatterjee, R et al 2008, ApJ, 689, 79

[2] Jorstad, S. et al 2010, Apj, 715, 362

[3] $\mathrm{M}^{\mathrm{c} H a r d y}$ I. et al, 2004, MNRAS, 348, 783.

[4] $\mathrm{M}^{\mathrm{c}}$ Hardy I. et al, 2006, Nature, 444, 730

[5] M $^{\mathrm{c}}$ Hardy I. et al, 2007, MNRAS, 375, 1521

[6] $\mathrm{M}^{\mathrm{c}}$ Hardy I. et al, in preparation

[7] Uttley, P. and $\mathrm{M}^{\mathrm{c}}$ Hardy I, 2001, MNRAS, 323, L26 